\title{
Knowledge based decision support system
}

\author{
Kyungyong Chung ${ }^{1} \cdot$ Raouf Boutaba $^{2} \cdot$ Salim Hariri $^{3}$
}

Published online: 14 November 2015

(c) Springer Science+Business Media New York 2015

Welcome to this special issue of the Information Technology and Management journal on knowledge based decision support systems. The main goal for this special issue is to be a timely vehicle for publishing selected research papers from academia and practitioners in different industries on this emerging topic.

Knowledge-based decision support systems are systems designed to ensure more precise decision-making by effectively using timely and appropriate data, information, and knowledge management for convergence industry. These systems refer to decision-making based on relevant knowledge, which is based on artificial intelligence, and on the application of information and communication technologies. In addition, these systems support decisionmaking through prediction and recommendation techniques. Depending on the criteria, there are various classifications. Based on the knowledge used for deduction, data is classified into knowledge-based systems using dictionary-defined knowledge, and non-knowledge-based

Kyungyong Chung

kyungyong.chung@gmail.com

Raouf Boutaba

rboutaba@uwaterloo.ca

Salim Hariri

hariri@ece.arizona.edu

1 Department of Computer Information Engineering, Sangji University, 83, Sangjidae-gil, Usan-dong, Wonju-si, Gangwon-do, Republic of Korea

2 David R. Cheriton School of Computer Science, University of Waterloo, 200 University Avenue West, Waterloo, ON N2L 3G1, Canada

3 NSF I/UCRC Center for Cloud and Autonomic Computing, University of Arizona, 1230 E. Speedway Blvd., Tucson, AZ 85721-0104, USA systems using machine learning and multi-dimensional statistical pattern recognition techniques [1-3]. This special issue covers some of the hottest topics in knowledge-based decision support systems, including: Decision Support for Convergence; Knowledge-based Applications and Management; Knowledge Acquisition and Representation; Knowledge Bases; Knowledge based Recommendation Systems; Data Modeling; Database Management Systems; Data Mining; Management Systems; Intelligent Healthcare Systems and Management; Decision Support Systems and Management; Machine Learning; Systems Analysis; and Design and Development.

The paper by Lee et al. [4] presents a clinical decision support system in medical knowledge literature reviews. They propose a method to increase performance and efficiency in a clinical decision support system (CDSS), and enhance the understanding of the CDSS for better health management among physicians and patients. To add structure to the current study, major research areas were categorized based on a multidimensional unfolding analysis. The academic outlook of medical informatics could be forecasted, and academic quality could be improved by addressing the problems arising out of system development and realization processes. The paper by Lee [5] presents factors influencing a social networking service (SNS) user's value perceptions, and word-of-mouth (WOM) decisions of corporate posts with special reference to emotional attachment. This study proposes a research model of the social knowledge value perception and WOM decision variable, including several precedent variables of a user's personal value factors, such as emotional attachment, self-esteem, and self-exposure. The SNS user recognizes the value of social knowledge through emotional and personal factors. 
The paper by Chung et al. [6] introduces a knowledgebased dietary nutrition recommendation system for obese management. This study proposes dietary nutrition recommendations for obese youth based on knowledge. The knowledge-based dietary nutrition recommendations herein include not only static dietary nutritional data but also individualized diet menus for them by utilizing knowledgebased context data through collaborative filtering. They developed knowledge-based dietary nutritional recommendation for obese management is expected to be effective in preventing obesity and preventing socioeconomic losses, as well. The paper by Lim et al. [7] presents a study on factor analysis to support knowledge-based decisions for a smart class. They propose to discover important elements that allow a smart class to achieve positive effects in education. The factors (ITLA system playfulness, perceived usefulness, perceived ease of use, attitude toward the class) that must be considered in the design and application of an effective smart class can be suggested to educators, researchers, developers, and education policy decision makers to support effective decisions.

The paper by Choi et al. [8] presents the common data model for a decision support system on adverse drug reactions (ADRs) to extract knowledge from a multi-center database. They propose that transformed data from an electronic medical record (EMR)-based ADR common data model (EADR CDM) is helpful in understanding prescription patterns and for exploring a feasible medication list for adverse drug signal detection. The collection of diverse data using a common data model is an effective method for early decisions on adverse drug reactions. The paper by Park et al. [9] presents a knowledge-based health service considering user convenience using hybrid $\mathrm{Wi}-\mathrm{Fi}$ peer-to-peer (P2P) networking. They propose a high-quality health service by building a network using a dispersed cross-layer optimization algorithm. The algorithm optimizes variables for the transmission control protocol/internet protocol (TCP/IP) stack in order to improve the energy efficiency and system reliability of a U-health sensor network. With real-time monitoring of context variables, the network can be applied to various conditions and requisites, and the proposed network configuration is expected to be easily integrated with existing wireless devices because the medium access control (MAC) layer is not defined.

The paper by Lee et al. [10] presents a knowledge-based freight management decision support system incorporating economies of scale with a multimodal minimum cost flow optimization approach. They propose multimodal minimum cost flow problem formulation with concave equations due to economies of scale for quantity, nonlinear equations due to economies of scale for both quantity and distance, and non-continuous equations due to economies of scale for vehicle size. This study explicitly considers several multimodal freight transport options in terms of quantity, vehicle size, batch strategy, multi-modes, and combinations. The paper by Han [11] presents efficient decision support for detecting content polluters on social networks with an approach based on automatic knowledge acquisition from behavioral patterns. This study is an efficient method for detecting content polluters on Twitter. They propose a set of features that can be easily extracted from the messages and behaviors of Twitter users, and constructs a new breed of classifiers based on these features.

The selection of papers has been achieved through careful evaluation and discussion of the papers. We gratefully acknowledge the authors for their worthy contributions to this special issue. We would also like to thank all members of the ICCT Technical Program Committee and the anonymous reviewers for their help in identifying original and innovative papers from the conference program and for their careful evaluation of earlier versions of the submitted papers. Accordingly, 8 high-quality papers were selected out of the 25 submitted resulting in a $32 \%$ acceptance rate. Last but not the least, we would like to thank Professor R.A. Patterson, Professor E. Rolland, coeditor in chiefs of the international journal on Information Technology and Management, for his valuable remarks and his undeterred help throughout the publication process of this special issue.

\section{References}

1. Chung K, Boutaba R, Hariri S (2014) Recent trends in digital convergence information system. Wirel Pers Commun 79(4):2409-2413

2. Kim SH, Chung K (2015) Emergency situation monitoring service using context motion tracking of chronic disease patients. Clust Comput 18(2):747-759

3. Kim JH, Ryu JK (2013) Recent trends on high-performance computing and security. Clust Comput 16(2):207-208

4. Yang J, Kang U, Lee Y (2015) Clinical decision support system in medical knowledge literature review. Inf Technol Manage. doi:10.1007/s10799-015-0216-6

5. Lee SH (2015) Factors influencing the social networking service user's value perception and word of mouth decision of corporate post with special reference to the emotional attachment. Inf Technol Manage. doi:10.1007/s10799-015-0227-3

6. Jung H, Chung K (2015) Knowledge-based dietary nutrition recommendation for obese management. Inf Technol Manage. doi:10.1007/s10799-015-0218-4

7. Jo J, Park J, Ji H, Yang Y, Lim H (2015) A study on factor analysis to support knowledge based decisions for a smart class. Inf Technol Manage. doi:10.1007/s10799-015-0222-8

8. Rho MJ, Kim SR, Park SH, Jang KS, Park BJ, Hong JY, Choi IY (2015) Common data model for decision support system of adverse drug reaction to extract knowledge from multi-center database. Inf Technol Manage. doi:10.1007/s10799-015-0240-6 
9. Chung K, Kim JC, Park RC (2015) Knowledge-based health service considering user convenience using hybrid Wi-Fi P2P. Inf Technol Manage. doi:10.1007/s10799-015-0241-5

10. Kim NS, Park B, Lee KD (2015) A knowledge based freight management decision support system incorporating economies of scale: multimodal minimum cost flow optimization approach. Inf Technol Manage. doi:10.1007/s10799-014-0209-x
11. Park BJ, Han JS (2015) Efficient decision support for detecting content polluters on social networks: an approach based on automatic knowledge acquisition from behavioral patterns. Inf Technol Manage. doi:10.1007/s10799-015-0250-4 